\title{
DESSEINS : LE DESSIN COMME POSTURE \\ PERFORMATIVE OÙ LA CONTEMPLATION \\ S'AVÈRE AUSSI UNE FORME D'ACTION
}

MATTE, HÉLÈNE.

Candidate au doctorat en littérature, arts de la scène et de l'écran à l'Université Laval

Résumé : Dessiner est un geste radical. Dans «Desseins : le dessin comme posture performative où la contemplation s'avère aussi une forme d'action ", l'artiste et chercheure Hélène Matte tente d'en faire la démonstration. Pour illustrer ses propos, elle s'appuie plus sur l'étymologie du mot et les écrits de Nietzsche sur la figure du contemplatif que sur ses esquisses réalisées en situation de désobéissance civile ou sur ses portraits de militants. Du « dessein » à la rêverie nietzschéenne, c'est la vivacité du processus qui détermine la valeur. Enfın, le médium dans son rapport au temps et sa comparaison à d'autres médiums sert aussi d'argument : tandis que la photographie institue une preuve, le dessin ne fait-il pas œuvre de témoignage?

Mots-clés : Dessin, performatif, performance, militant, Nietzsche

\begin{abstract}
Drawing is a radical gesture. In "Desseins : le dessin comme posture performative où la contemplation s'avère aussi une forme d'action", artist and researcher Hélène Matte is demonstrating it. To illustrate her intentions, she leans on the etymology of the word and on Nietzsche's writings on the contemplative, as well as on her portraits of activists and on her own drawings realized in situations of civil disobedience. From "dessein" to nietzschean reverie, it is the liveliness of the process that determines its value. Finally, the medium, in relation to time and in comparison to other mediums, also serves as argument: while photography leaves proof, doesn't the drawing act as witness?
\end{abstract}

Keywords : Drawing, performative, performance, activist, Nietzsche 
Des images dans l'œil humain! Voilà ce qui domine tout être humain : à partir de l'œeil!

Sujet! l'oreille entend le son! Une tout autre conception, merveilleuse du même monde.

Nietzsche, Euvres

Le postulat que je présente constitue aussi le titre de cet article. Il importe de situer d'abord le performatif dont il est question. En fait, l'usage de ce terme renvoie aux théories du langage et à ce que la pragmatique linguistique a nommé, après les travaux d'Austin, les énonciations performatives. Ma posture renverse en un premier temps le « dire c'est faire » austinien en « faire c'est dire ». Puisqu'il s'agit ici d'une pratique du dessin, le « oui je le veux » de l'engagement n'est pas illocutoire ; il s'agit d'un acte de langage non-verbal, d'un geste avant la parole. Aussi, le « oui » qu'il nécessite n'est pas celui que porte une volonté, mais celui qu'imposent les contingences. Toutefois, si Austin dénonce «l'illusion descriptive » du langage pour lui opposer une fonction agissante, c'est dans une même optique que le dessin d'observation est ici abordé. En l'occurrence, la performativité dont il est question appelle la Performance entendue comme discipline artistique. II s'agit de la Performance au sens où Rose Lee Goldberg la décrit comme une " présence ésotérique, chamanique, pédagogique, provocatrice ou divertissante » (Goldberg, 2001 : 8). Dans cette perspective, l'acte de dessiner concerne le corps ainsi que les rapports entre les corps individuels et sociaux.

\section{L'EXPÉRIENCE DU DESSIN}

Le dessin est au cœur de ma pratique, une pratique plurielle passant de la poésie orale à la performance sans texte jusqu'à l'image sur papier ou toile. Mon dessin' est principalement basé sur l'observation. Je reproduis et interprète des images mais principalement, ce sont des objets, des scènes et des personnes réels, à mes yeux, que j'esquisse et crayonne. Depuis longtemps, j'ai l'habitude de saisir au passage les lieux et les gens. En voyage, le dessin a été privilégié aux dépends des photos-souvenirs. Au restaurant, à la piscine, dans les salles d'attente, lorsque j'assiste à des concerts, même au cinéma : toujours le dessin m'a permis de saisir mon environnement, de faire la chronique du quotidien. Aujourd'hui, je présente plus de soixante dessins réalisés au cours des dernières années. La plupart de ceux qui représentent des manifestations ou des actions de désobéissance civile datent de plus d'une dizaine d'années, à une période où je m'occupais principalement à militer et à dessiner, les deux en même temps si possible. Ces dessins ont été réalisés sur le vif. Certains ont été retouchés après les évènements.

En public, la personne se consacrant au dessin d'observation se découvre en situation paradoxale. Elle s'exclut de la collectivité pour se concentrer sur sa tâche et devenir elle-même objet d'observation. De plus, elle entre en contact privilégié avec l'espace et les autres individus qui s'y trouvent. Lorsqu'il s'agit de dessiner un portrait, l'aspect relationnel devient l'enjeu du dessin et donne lieu à de véritables rencontres, à d'intenses échanges de regards, à des conversations et parfois à des confidences. De même, le dessin réalisé lors de circonstances où il n'est pas attendu fait évène-

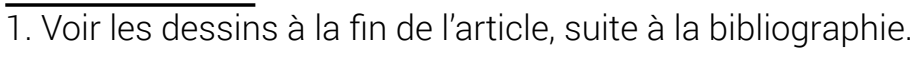


ment. II s'agit alors de s'adapter plus que d'ajuster l'environnement à sa guise, de saisir un mouvement continu plutôt que de fixer une image. Plus que le sens de la vue, le dessin d'observation implique l'entièreté du corps. Que ce soit en pleine rue ou en haut d'une grue lors d'une occupation, il m'ouvre à l'espace que j'habite. Le dessin est ainsi davantage un acte performatif. Plus qu'un point de vue, il se fait corps et geste.

\section{SENS ET REPRÉSENTATION}

L'acte photographique et vidéographique n'est pas neutre. Malgré son " objectif », sa fonction est souvent ostentatoire. J'ai décidé de ne pas prendre de photos pour documenter les actions, mais plutôt de dessiner, de répondre à l'action par l'action, de dialoguer par ce que le geste a d'unique. Ainsi je dessine les musiciens, les artistes audio et les performeurs. Plus qu'une écriture, le dessin d'observation est aussi un mode de lecture. II est réception et interprétation. Du regard à la main, de la main au crayon, du crayon à l'encre, de l'encre au papier : le dessin d'observation est une traversée. II aplatit au sein de la page la géométrie des lieux et des visages. II abandonne ou révèle certains détails. II condense une totalité passagère.

Dessin, c'est aussi « dessein », du mot italien disegno, à la fois marque d'un acte passé et direction d'avenir. Il est sens :

En passant par la langue française, le mot conserva son double sens, matériel et intellectuel, de tracé et de projet... Expression visible d'une idée, d'une représentation mentale où sont convoqués le langage, le corps, l'espace et le temps. Le dessin fut encore envisagé comme écriture vectorisant l'espace de la feuille, de la toile, du lieu où et d'où l'on écrit... Dessein perdra son « e » pour devenir dessin vers le XVIIIle siècle. L'aspect "projet" sera mis à l'écart et sa définition sera réduite à une dimension pratique. (Lichtenstein, $2007:$ 19)

L'antécédent du dessin nous rappelle d'où l'on vient : le trait et le cercle, la ligne et la forme comme première écriture. Une archi-écriture dont le sens est marque de passage. Une écriture qui implique plus qu'elle n'explique. Aussi, le dessin d'observation nous indique où l'on va : au cœur des modulations de notre perception du temps et de l'espace, devant les regards que nous côtoyons. Esquisse, manuscrit ou plan précurseur : le dessin était jadis à la base de toute pratique sans être une pratique à lui seul. Qui plus est, il porte jusque dans son étymologie le mouvement d'émergence à l'œuvre, de l'œuvre. II est processus.

En dessinant, je ne cherche pas nécessairement à représenter l'objet; j'entreprends de le percevoir. Je peux ainsi traduire, dans l'instant de la réception, une action accomplie devant moi ou encore l'action que je pose moi-même. Car il m'est déjà apparu, non pas en tant qu'artiste ou spectatrice, mais en tant que militante, que le dessin était plus pertinent que la photographie lors d'actions de désobéissance civile. L'économie des moyens et l'anonymat s'imposent alors, particulièrement en situation d'action directe. Tandis que la photographie institue une preuve, le dessin, quant à lui, fait œuvre de témoignage. De même, le dessin radical et militant n'est pas celui des caricaturistes, dont les péripéties des dernières années ont certes démontré à quel point leur geste n'est pas anodin et rappellent qu'il y a une échelle de valeurs vertigineuses dans les rapports à la représentation. 
L'image n'est jamais neutre. Or, le dessin dont il est question n'est pas non plus celui qui invente les idéogrammes de la pensée militante, des logos du discours engagé. II n'est pas plus celui qui contribue à l'imagerie des affiches et des publications entourant une cause sociale, celle des étudiants contre la hausse des frais de scolarité par exemple, pour laquellej'ai réalisé nombreux tracts et affiches. Ce dessin d'observation n'est pas celui qui se contenterait de portraiturer de grandes figures du militantisme : du mouvement syndical, comme Michel Chartrand, ou de l'émancipation des Noirs, tel un Black Panther. Ce n'est pas davantage, par des représentations misérabilistes ou excessivement optimistes, peindre spectaculairement la misère pour dénoncer les catastrophes, ou, à l'inverse, glorifier des luttes historiques édifiantes.

Le dessin dont il est question est celui qui est fait dans l'instant afin d'en saisir l'urgence. Paradoxalement, il transforme le rapport au temps, il en ralentit la cadence. II désynchronise la réception, il déplace le lieu de l'évènement. Ce dessin brise l'unicité des représentations. II s'expose au risque corporel et matériel de son exécution. C'est celui dans lequel chaque trait est crucial et chaque lumière, significative. Pour l'intelligence sensible, le dessin d'observation permet, à l'époque des processus, des modalités communicationnelles et des traductions effrénées, de méditer sur l'agitation des gens et des lieux. À l'ère de la reproductibilité technique et de la marchandisation du nombre, il implique le geste et son exception. II ouvre à la matière sensible de manière à ce que l'action ne soit pas seulement motivée par une idéologie, mais qu'elle participe aussi à une expérience sensuelle et sentie. L'objectif militant n'est alors pas seulement la visée mécanique et logique d'un but qu'est le changement de politique. Par ses aspirations, l'engagement est un regard porté par le désir, il est par lui-même transformations et sensibilités.

\section{CONTEMPLER, FAIRE ET DÉFAIRE}

À l'instar de Nietzsche dans la citation suivante, je réfute la scission dialectique qu'il est d'usage d'établir entre la contemplation et l'action. Pour Nietzsche, la vie contemplative est celle qui est susceptible de créer de nouvelles valeurs. Par conséquent, elle bénéficie d'un pouvoir de transformation :

[Le contemplatif] possède certainement en propre, en tant que poète, la vis contemplativa et le regard rétrospectif sur son œuvre, mais en même temps et au premier chef, la vis creativa qui manque à l'homme d'action, en dépit de l'apparence et de la croyance commune. C'est nous, les hommes qui sentent en pensant, qui ne cessons de construire réellement quelque chose qui n'existe pas encore : tout le monde éternellement en croissance des appréciations, des couleurs, des poids, des perspectives, des gradations, des acquiescements et des négations. (Nietzsche, [1882] 2000 : 301)

Considérant la posture performative du dessin comme il m'est arrivé de le pratiquer, j'affirme que la contemplation s'avère aussi une forme d'action.

Pour conclure, je me permets une digression et convoque à nouveau Austin et sa célèbre formule « dire c'est faire ». Je ne renonce pas pour autant à l'« illusion descriptive » présentée en introduction ; au contraire, je me prête au jeu et me prends aux mots. J'imagine esquisser le lieu même où se donne à entendre cette communication. Mon dessin est alors une mise en abyme 
du colloque Action radicale, sujet radical auquel je participe en tant que doctorante. II fait écho aux discussions sur l'éducation et sur le Printemps érable qui ont animé les premières séances. De ma chaise confortable, je dessine une salle grise au centre de laquelle une longue table est criblée de micros. Je remarque en dessinant qu'il y a un micro pour chaque communicant, mais aucune lumière d'appoint. Je constate l'impossibilité d'éteindre les lampes complètement pour avoir une visibilité maximale à l'écran sans compromettre la lecture ou la prise de notes. Ainsi, les technologies du son ont bien été réfléchies à cet endroit. Cependant, aucune réflexion sur l'éclairage n'est manifeste. Je ne peux pas dessiner les ondes électromagnétiques qui se mêlent aux vibrations du projecteur ni les ombres. II n'y a pas d'ombre. Les néons au plafond offrent peu de contraste et tout est plat, malgré la perspective de la pièce.

Que dire de cette salle de conférence partiellement fonctionnelle au Carrefour des arts et des sciences de l'Université de Montréal? Illustre-t-elle notre rapport actuel au savoir? Cette armée de micros est-elle l'indice d'un certain phonocentrisme, au sens où Derrida l'associe à un logocentrisme réduisant l'écriture à un supplément de parole (Derrida, 1967: 16)? Est-elle simplement l'œuvre d'une technocratie tenant pour acquis que nous n'avons plus assez de souffle pour faire entendre nos voix? Cette lumière industrielle, digne d'un entrepôt, d'un centre d'achat ou pire, d'un abattoir, n'offense-t-elle pas les « lumières », au sens figuré? Ne nous éclaire-t-elle pas sur l'achèvement d'une approche humaniste de l'éducation au profit d'une marchandisation du savoir? Heureusement, dans le décor de ces tristes métaphores s'animent les étudiants et les professeurs. Dans mon dessin, ils brisent les lignes vectorielles modelant l'étroitesse du lieu sans fenêtre. J'ébauche cette communauté ponctuelle, j'y trouve des regards et des horizons qui se fusionnent : des architectures de pensées, les dérives ou les déconstructions qu'elles appellent. 
Derrida, Jacques. De la grammatologie. Paris : Minuit, 1967. Imprimé.

Goldberg, Rose Lee. La Performance, du futurisme à nos jours. Paris: Thames \& Hudson, 2001. Imprimé. Langhshaw Austin, John. Quand dire c’est faire. Paris : Seuil, 1970. Imprimé.

Lichtenstein, Jacqueline, dir. Du disegno au dessin. Du Dessin au dessein. Bruxelles : La Lettre volée, 2007. Imprimé.

Nietzsche, Friedrich. Le Gai savoir. 1882. Paris : Flammarion, 2000. Imprimé.

---. Euvres philosophiques complètes II. Paris: Gallimard, 1988. Imprimé. 


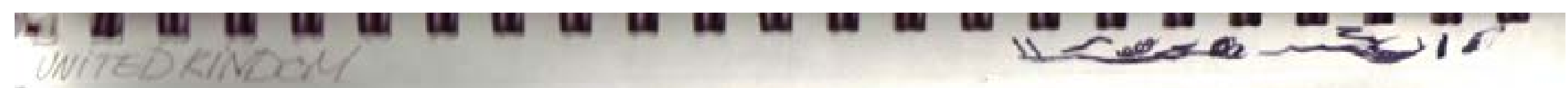

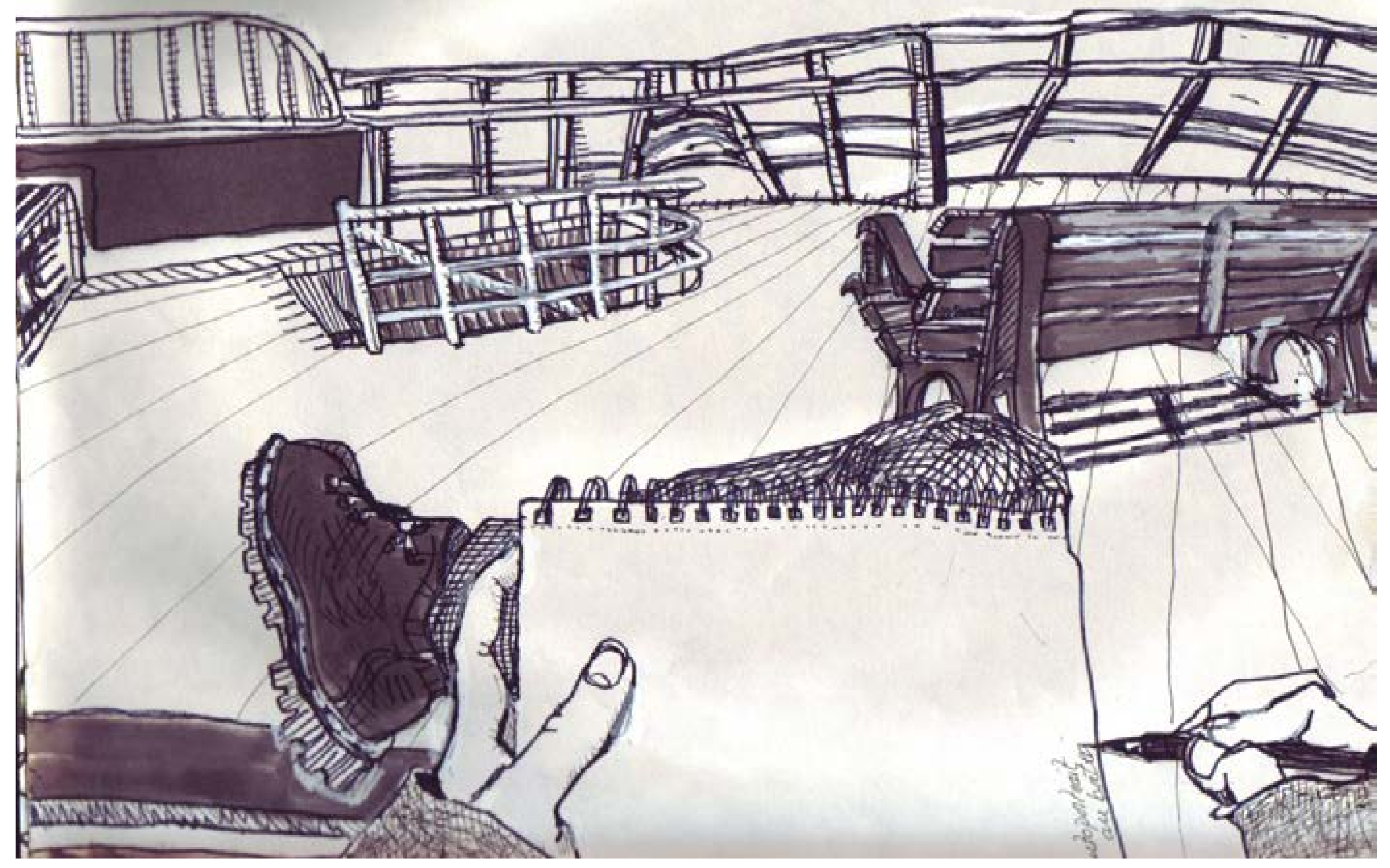




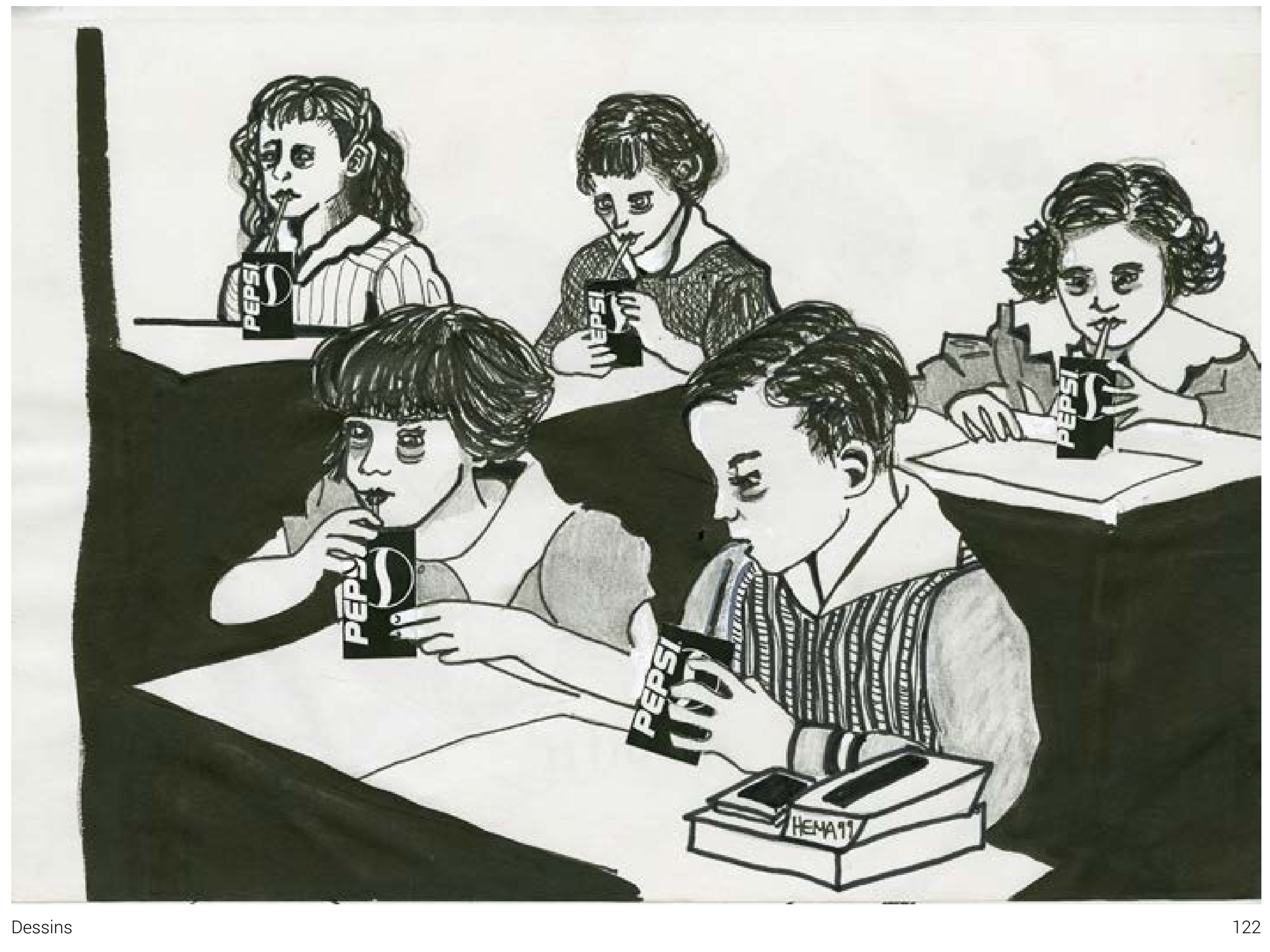




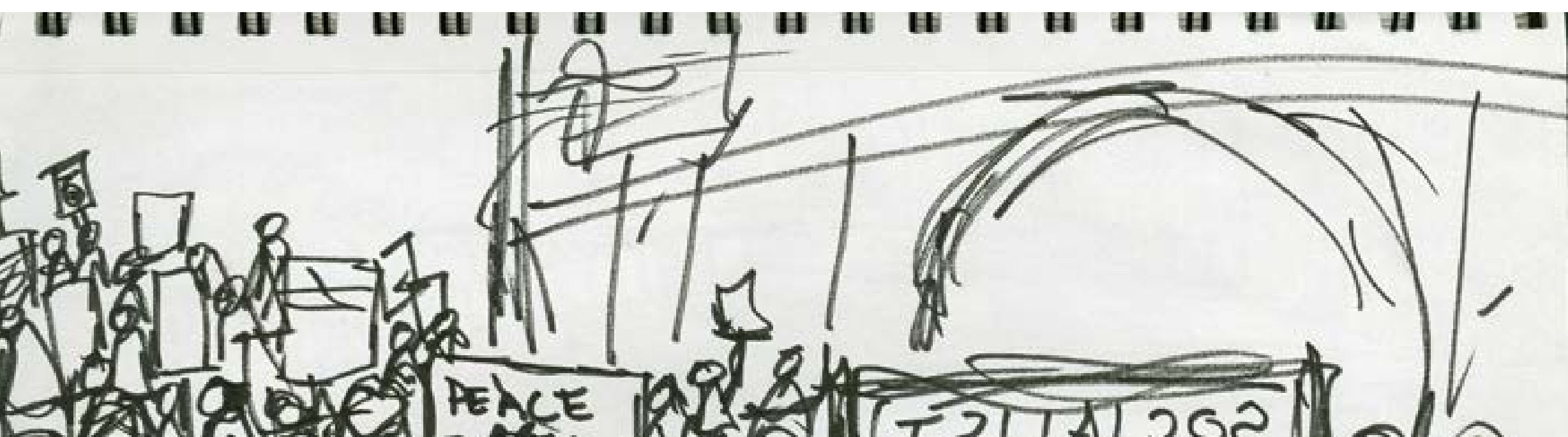

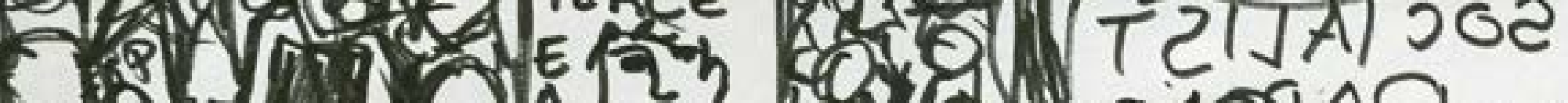

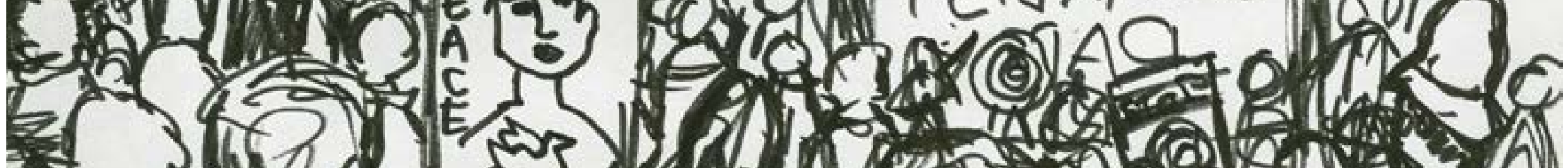

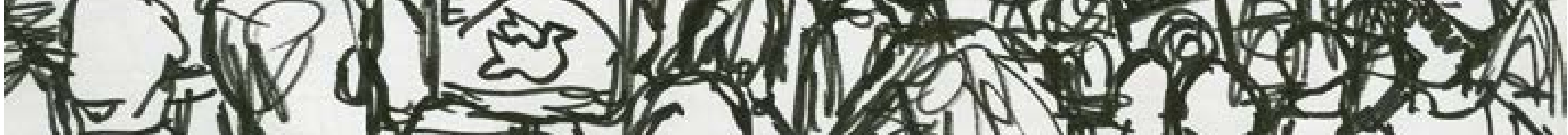
Not 1 (

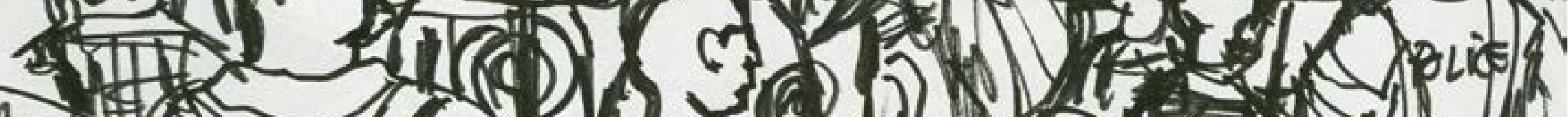

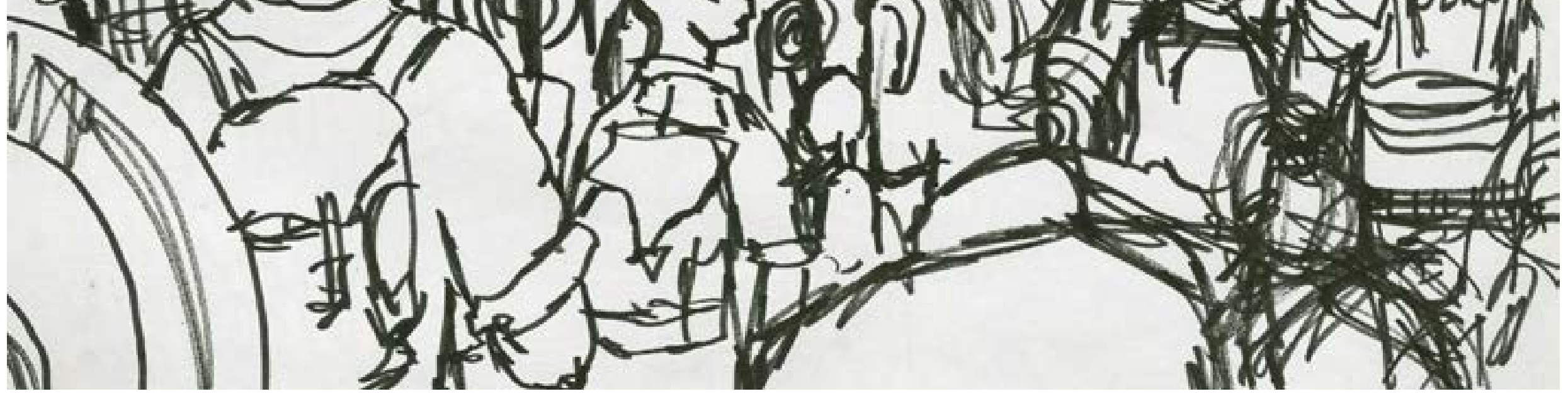




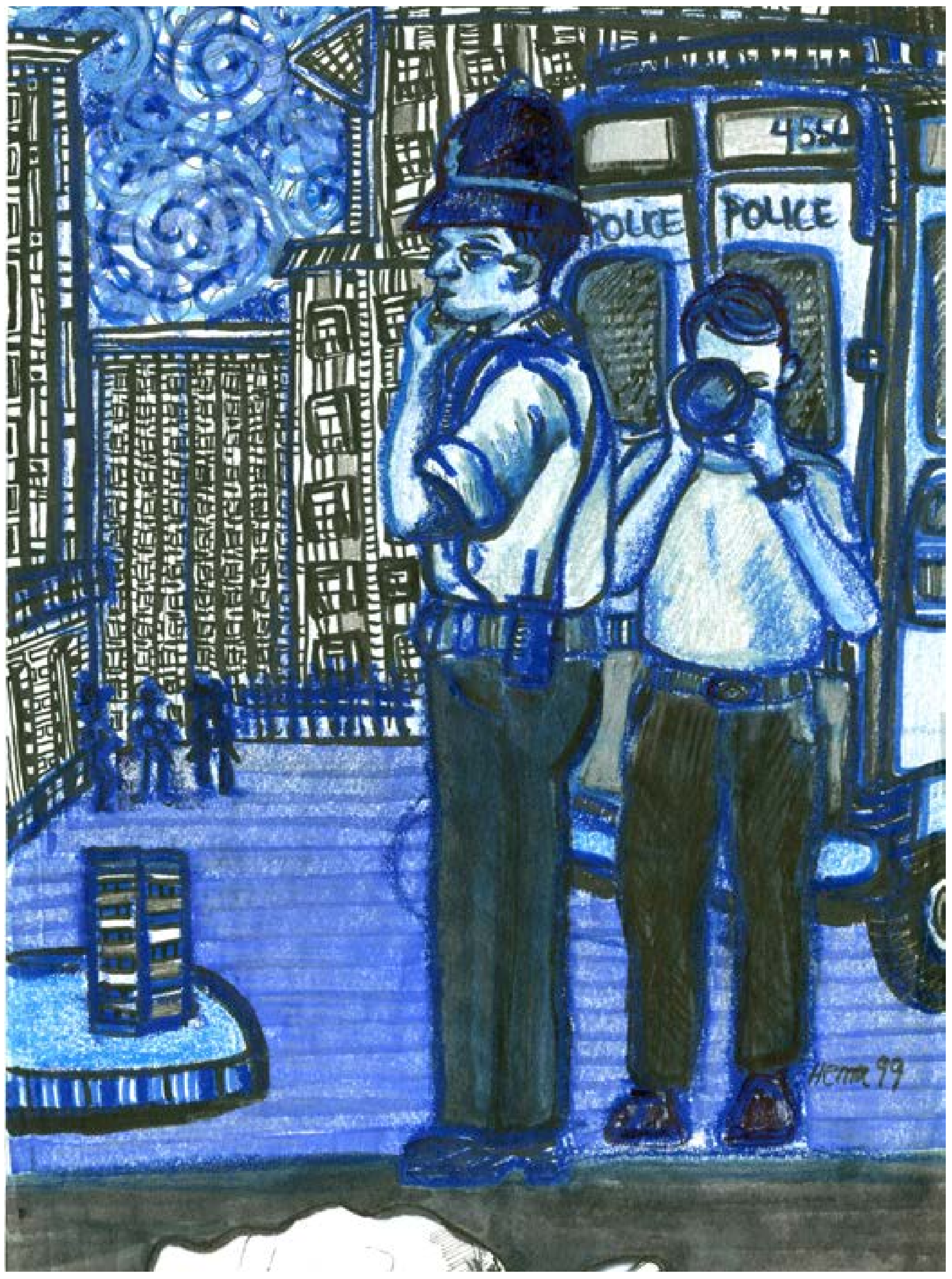




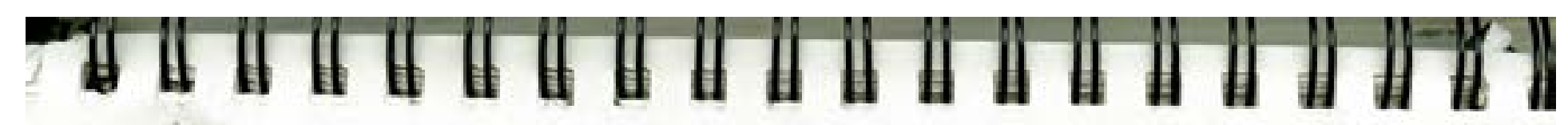

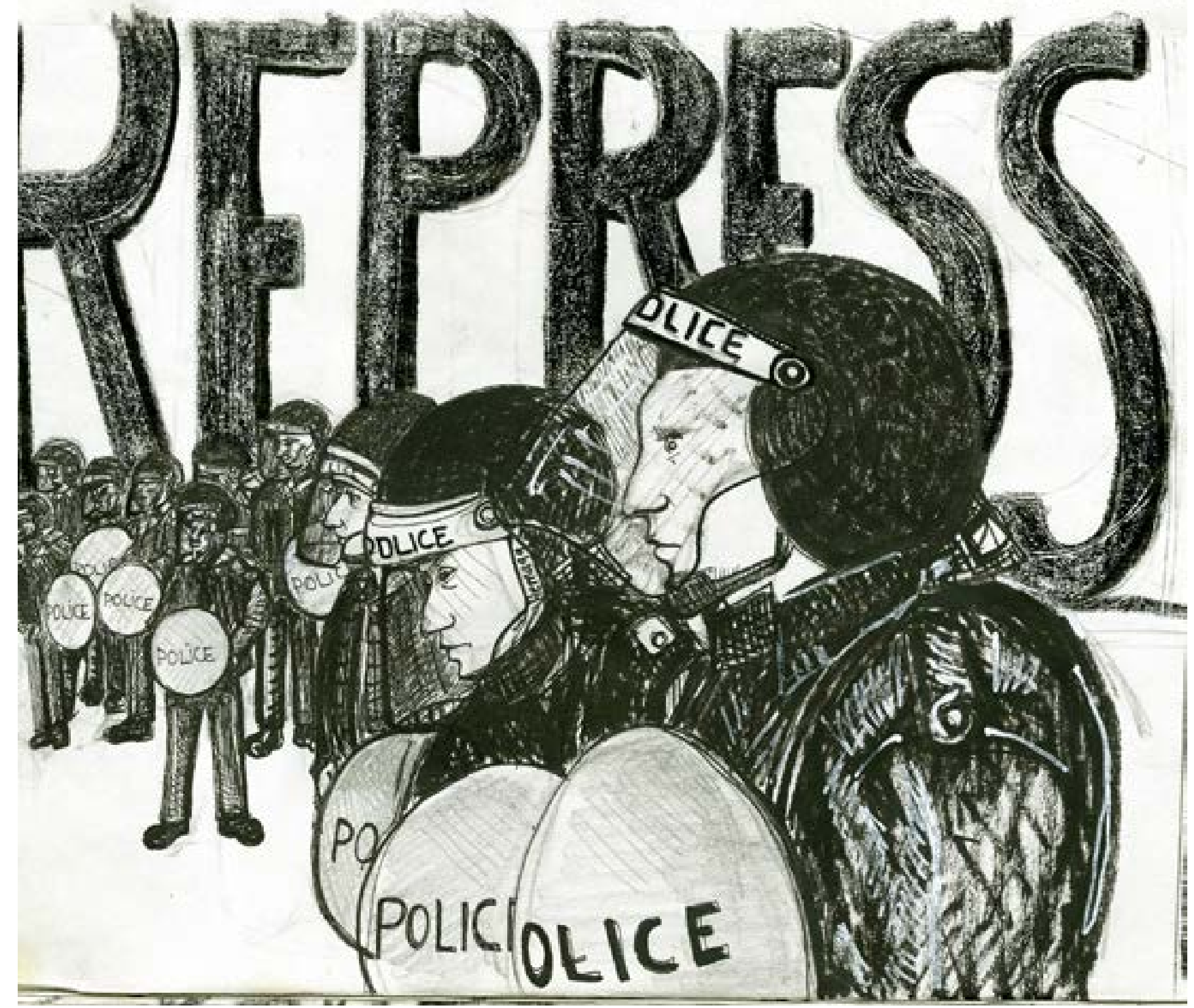




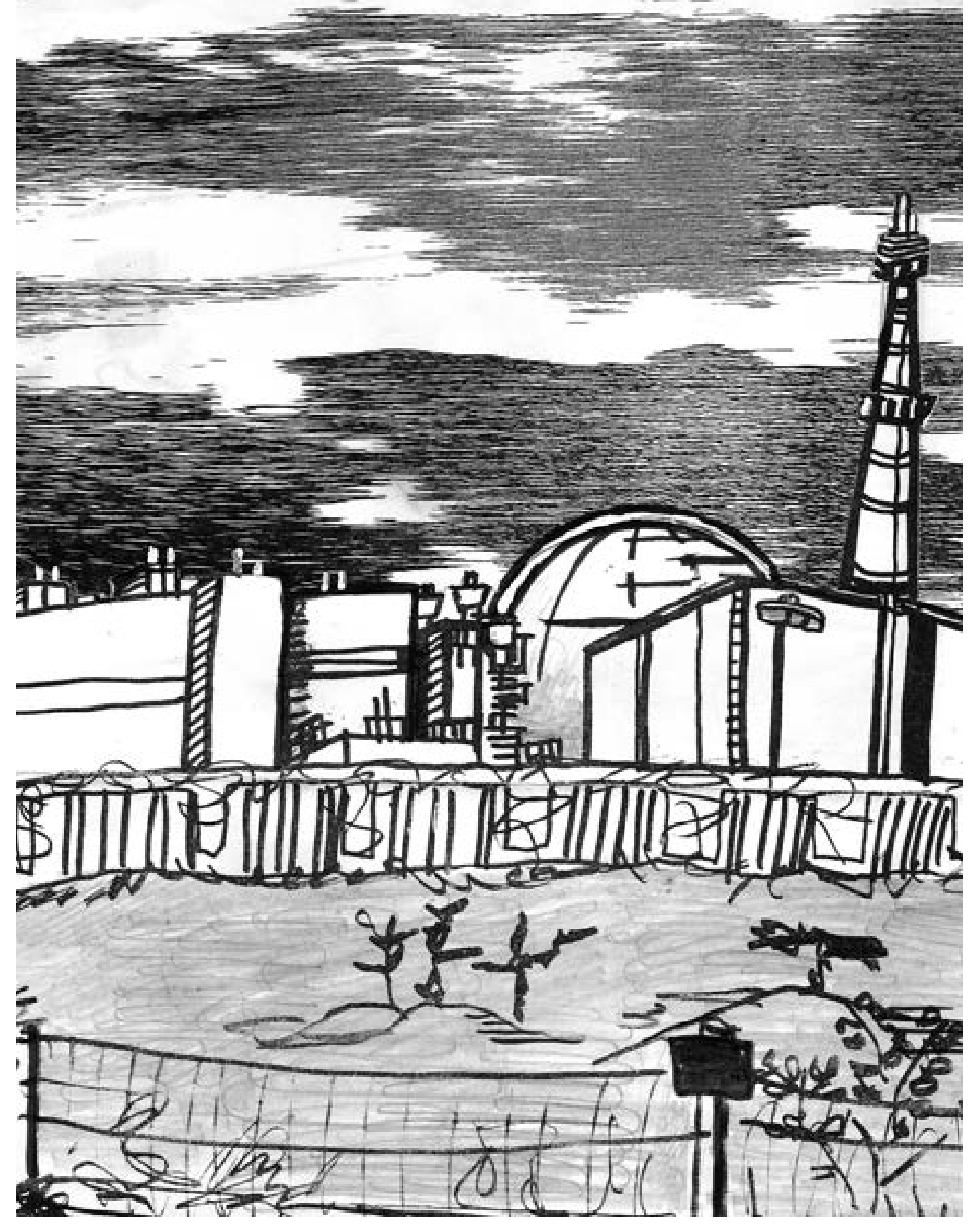




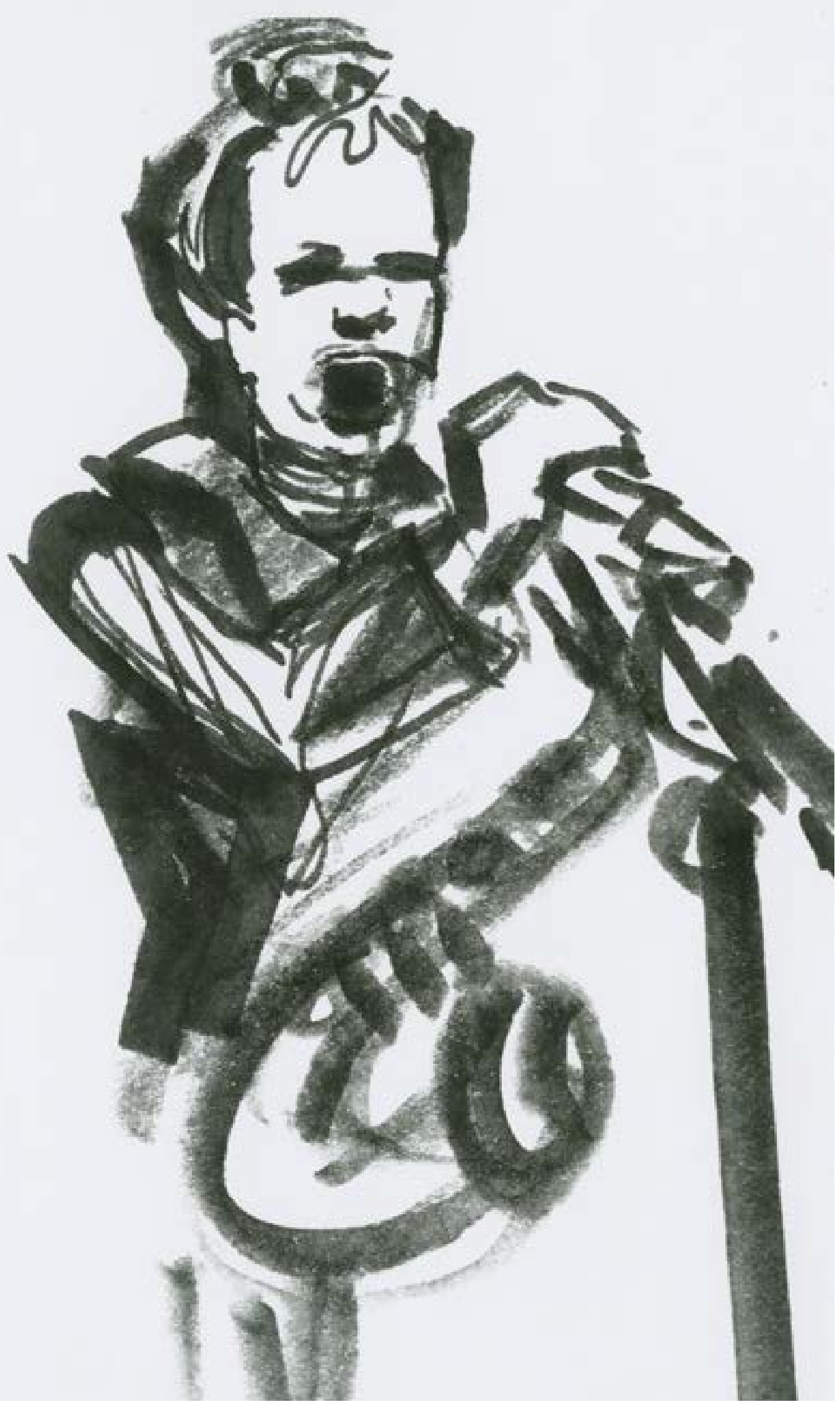

